Seventh Meeting; May 10th, 1895.

Wy. Peddie, Esq., M.A., D.Sc., in the Chair.

\title{
Proof of a Theorem in Conics.
}

By R. F. Muirhead, M.A.

I.

In text books of Plane Coordinate Geometry, two methods are usually given for investigating the condition that the general equation of the second degree:

$$
\phi \equiv a x^{2}+b y^{2}+c z^{2}+2 f y z+2 g z x+2 h x y=0
$$

may represent a pair of real or imaginary straight lines.

The first is by identifying $\phi$ with the product of two linear factors, say $\quad \lambda \lambda^{\prime} \equiv(l x+m y+n z)\left(l^{\prime} x+m^{\prime} y+n^{\prime} z\right)$.

Equating coefficients, and eliminating $l, m, n, l^{\prime}, m^{\prime}, n^{\prime}$, we get

$$
\left|\begin{array}{lll}
a & h & g \\
h & b & f \\
g & f & c
\end{array}\right|=0, \text { or, Discriminant }=0
$$

as the condition required.

The second method consists in solving $\phi=0$ as a quadratic equation in $x$, and deducing the condition that the expression in $y$ and $z$ under the radical sign, should be a perfect square.

This as before, gives the condition : Discriminant $=0$.

We may note by the way that of these two methods, the former, strictly speaking, proves only the necessity, and the latter, only the sufficiency of the condition; so that the propositions proved are converse, one of the other. 
The object of this Note is to point out a short way of performing the elimination required in the former method, by forming the determinant which is the product of the two zero determinants

$$
\left|\begin{array}{c}
l, l^{\prime}, o \\
m, m^{\prime}, o \\
n, n^{\prime}, o
\end{array}\right| \text { and }\left|\begin{array}{c}
l^{\prime}, l, o \\
m^{\prime}, m, o \\
n^{\prime}, n, o
\end{array}\right|
$$

The product is the symmetrical determinant

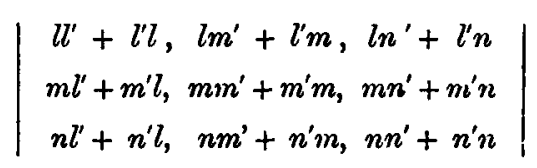

which is of course identically equal to zero.

But if $\phi$ is identical with $\lambda \lambda^{\prime}$ the determinant is obviously the same as

$$
8 \times\left|\begin{array}{ccc}
a & h & g \\
h & b & f \\
g & f & c
\end{array}\right|
$$

Thus the discriminant of $\phi$ is zero if $\phi$ represents a pair of straight lines.

Of course $\lambda \lambda^{\prime}=0$ is the standard form when we have a pair of real straight lines; and can only represent an imaginary pair when some of the coefficients are imaginary. The standard form for a pair of imaginary lines (or point-ellipse) would be $\lambda^{2}+\lambda^{\prime 2}=0$, where $\lambda \equiv l x+m y+n z$, etc.

In this case the identification with $\phi$ gives

$$
a=l^{2}+l^{\prime 2}, f=m 2 n+m^{\prime} n^{\prime}, \text { etc., etc. }
$$

And the elimination of $l, m, n, l^{\prime}, m^{\prime}, n^{\prime}$ can here be performed by squaring the zero determinant

$$
\left|\begin{array}{lll}
l & l & o \\
m & m^{\prime} & o \\
n & n^{\prime} & o
\end{array}\right|
$$

and substituting $a$ for $l^{2}+l^{\prime \prime}, f$ for $m n+m^{\prime} n^{\prime}$, etc., in the result. 
II.

It occurred to me recently that this method of getting the condition discriminant $=0$ by multiplying two determinants, might be capable of application to discuss the discriminant in the general case. I have only had leisure to make a beginning in this direction, and none to look up the literature of the subject; but the following results seem interesting, and are new to me.

Suppose the general expression $\phi$ put into the form

$$
p \lambda^{2}+p^{\prime} \lambda^{\prime 2}+p^{\prime \prime} \lambda^{\prime \prime 2}
$$

where $p p^{\prime} p^{\prime \prime}$ are constants and $\lambda \equiv l x+m y+n z$, etc. ; thus we have

$$
a=p l^{2}+p^{\prime} l^{2}+p^{\prime \prime} l^{\prime 2}, f=p m n+p^{\prime} m^{\prime} n^{\prime}+p^{\prime \prime} m^{\prime \prime} n^{\prime \prime}, \text { etc., etc. }
$$

and the discriminant

$$
\left|\begin{array}{lll}
a & h & g \\
h & i & f \\
g & f & c
\end{array}\right|
$$

is obviously

the product

$$
\left|\begin{array}{ccc}
l & l^{\prime} & l^{\prime \prime} \\
m & m^{\prime} & m^{\prime \prime} \\
n & n^{\prime} & n^{\prime \prime}
\end{array}\right| \times\left|\begin{array}{ccc}
p l, & p^{\prime} l^{\prime}, & p^{\prime \prime} l^{\prime \prime} \\
p m, & p^{\prime} m^{\prime}, & p^{\prime \prime} m^{\prime \prime} \\
p n, & p^{\prime} n^{\prime}, & p^{\prime \prime} n^{\prime \prime}
\end{array}\right|
$$

which may be written

$$
p p^{\prime} p^{\prime \prime} \times\left|\begin{array}{ccc}
l & l^{\prime} & l^{\prime \prime} \\
m & m^{\prime} & m^{\prime \prime} \\
n & n^{\prime} & n^{\prime \prime}
\end{array}\right|^{2}
$$

and this is $=p \cdot p^{\prime} \cdot p^{\prime \prime} . \mathrm{NN}^{\prime} \mathrm{N}^{\prime \prime} \times$ twice area of triangle formed by the lines $\lambda=0, \lambda^{\prime}=0, \lambda^{\prime \prime}=0$; where $N, N^{\prime}, N^{\prime \prime}$, are the minors of $n, n^{\prime}, n^{\prime \prime}$.

This of course vanishes when the lines are concurrent, in which case $\phi$ is expressible as the sum of two squared linear terms; and also when $p p^{\prime} p^{\prime \prime}=0$, i.e. when one at least of the squared terms is awanting.

The lines $\lambda=0, \lambda^{\prime}=0, \lambda^{\prime \prime}=0$ form a self-conjugate triangle for the conic; and such triangles are triply infinite in number for a given conic. We get the same result as to the possible number of ways of expressing $\phi$ in the form $p \lambda^{2}+p^{\prime} \lambda^{\prime 2}+p^{\prime \prime} \lambda^{\prime \prime 2}$ by noting that 
there are 8 independent ratios between the coefficients of the latter expression, and only 5 in $\phi$.

Again, it appears that the discriminant may vanish in virtue of $p^{\prime \prime}$ being zero, in which case the value of $\lambda^{\prime \prime}$ might be anything whatever ; in fact, it seems that in such a case, while two sides of a self-conjugate triangle must pass through the centre of the conic, the position of the third is quite indeterminate, a result which is obvious also from the geometrical point of view. 